\title{
Posterior Reversible Leukoencephalopathy Syndrome (PRES) in Intensive Care Unit - Case series
}

\author{
Antara Gokhale, Atheel Kimona1, Sandeep Kantor, S. Prakash, Yogesh Manhas \\ Departments of Anesthesia and Adult ICU and ${ }^{1}$ Radiology, Royal Hospital, Muscat, Oman
}

\section{Abstract}

Introduction: Posterior reversible encephalopathy syndrome (PRES) is characterized by a range of clinico neuro radiological manifestation along with hypertension due to varied etiology. Contrary to its name, a small number of patients have residual neurological deficits. Patients with severe manifestation of PRES may get admitted to the Intensive Care Unit (ICU) due to coma or status epilepticus. Small case series and large multicenter studies have been reported. Materials and Methods: We report a single center series of ten cases admitted to ICU over a 5 year period in a tertiary care centre. We retrospectively analyzed patients, admitted to adult ICU with different etiologies, and diagnosed to have PRES. Outcome at discharge and at 90 days after ICU admission was noted. Result: The most common etiologies were medications. ICU admissions were mainly for uncontrolled hypertension, seizures, and low level of consciousness. All except one patient were ventilated. Four patients had residual neurological damage. Conclusion: Irrespective of the etiology, early control of blood pressure, and removal of precipitating factors is of paramount importance to prevent morbidity and mortality. Awareness about PRES among the intensivist is necessary to ensure early recognition and treatment.

Keywords: ICU, PRES, Neuro-imaging

\section{InTRODUCTION}

Posterior reversible encephalopathy syndrome (PRES) is a clinco-radiological diagnosis characterized by acute and varied neurological manifestations along with magnetic resonance image (MRI) findings comprising of hyperintensities on T2-weighted images.

Widely described since 1996, the exact pathophysiology remains unknown. ${ }^{[1]}$ As the name suggests, initially it was described to involve the areas of posterior circulation of the brain; however, subsequently, other areas of the brain have been found to be affected [Figure 1]. About 15\%-20\% of patients have residual neurological deficits, and the syndrome is not always totally reversible. ${ }^{[2]}$

PRES has been described in people with varied clinical etiologies, and the list is ever increasing. Female predominance is seen. ${ }^{[1]}$ Around $35 \%-40 \%$ of patients need mechanical ventilation due to the severity of neurological symptoms and reach the Intensive Care Unit (ICU). ${ }^{[2,3]}$ Epidemiological data for patients admitted in ICU is lacking. We retrospectively analyzed patients admitted to a tertiary care ICU with varied etiologies and diagnosed with PRES over the duration of 5 years.

\begin{tabular}{|l|l|}
\hline \multicolumn{3}{c|}{ Access this article online } \\
\hline Quick Response Code: & Website: \\
& www.ijccm.org \\
& \\
\end{tabular}

A high degree of suspicion for early diagnosis and initiation of appropriate treatment is necessary to decrease morbidity and mortality. Treatments include control of blood pressure and remove the causative agent.

\section{Patients and Methods}

Patients admitted to an adult ICU of a tertiary care unit and diagnosed to have PRES over a 5-year period from 2010 to 2015 were retrospectively analyzed.

Clinical presentation, background medical history, and associated risk factors were reviewed. We documented the neurological status at presentation, namely, the presence of headache, seizures, status epilepticus, and other neurological manifestations. The need for mechanical ventilation, duration of ventilation, and outcome at the time of discharge from ICU was noted. Computerized tomograms (CT) and MRI

Address for correspondence: Dr. Antara Gokhale Department of Anesthesia and Adult ICU, Royal Hospital, P. 0. Box 1076, Azaiba Postal Code 130, Muscat, Oman. E-mail: gokhaleantara@gmail.com

This is an open access article distributed under the terms of the Creative Commons Attribution-NonCommercial-ShareAlike 3.0 License, which allows others to remix, tweak, and build upon the work non-commercially, as long as the author is credited and the new creations are licensed under the identical terms.

For reprints contact: reprints@medknow.com

How to cite this article: Gokhale A, Kimona A, Kantor S, Prakash S, Manhas Y. Posterior reversible leukoencephalopathy syndrome (PRES) in intensive care unit - Case series. Indian J Crit Care Med 2017;21:772-8. 
were reviewed to document the areas involved. Patient's neurological status and residual deficits if any at the time of discharge from the unit and after 90 days period were noted. The possible etiological factors were identified.

\section{RESULTS}

Over a 5-year period from 2010 to 2015 , we identified ten patients diagnosed with PRES [Table 1]. Out of these patients, four patients had systemic lupus erythematosus (SLE), one newly diagnosed, four had eclampsia of pregnancy, one patient of sickle cell disease with acute chest syndrome, and one with induction chemotherapy for acute lymphoid leukemia [Figures 2 and 3].

Female predominance (male:female-2:8) was seen with age range from 14 to 42 years. Accelerated hypertension was present in nine patients with average blood pressure of $200 / 100 \mathrm{mmHg}$. One of the patients had normal blood pressure. All of the patients except one presented with seizures and two patients had status epilepticus. All of the patients complained of headache and three had decreased level of consciousness. Nine patients required mechanical ventilation mainly for airway protection. One patient had repeat clinical and radiological features of PRES after recovery from the first episode within the 90 -day period. All patients except one had more than one areas of the brain involved.

Patients were ventilated for 3-15 days and the duration of ventilation was related to underlying etiology and control of the blood pressure. Patients with PRES secondary to eclampsia recovered faster as compared to those with background of chronic hypertension. Two patients with SLE continued to have seizures at the end of 3 months. The MRI in one of the patient showed changes suggestive of vasculitis and the other had new white matter lesions as a part of progression of the disease.

One patient with eclampsia presented with frontal infarction and continued to have left lower leg weakness with a limping gait at the end of 3 months. At the time of ICU discharge, two patients required tracheostomy for weaning from mechanical ventilation secondary to critical illness myoneuropathy and one had cognition impairment.

We lost one patient at the end of 90 days due to complicated Gram-negative septicemia and not related directly to PRES.

All patients with underlying autoimmune disease had chronic hypertension, renal involvement, and were on immunosuppression. Three patients developed PRES after receiving immunosuppression agent that was administered for flare-up of the disease.

As mentioned earlier, one patient developed PRES for the second time 1 month after clinical and radiological recovery from the initial episode, but it is not included as a separate case.

CT scan was the primary imaging done in nine patients and was normal in five. MRI was done in all patients and diagnosis of PRES was established [Table 2].

\section{Discussion}

PRES is a neurotoxic state that presents with varied neurological manifestations such as headache, seizures, encephalopathy, visual disturbance, and less commonly with focal neurological deficits. ${ }^{[2,3]}$ Seizures are seen in $92 \%$ of cases $^{[2]}$ with status epilepticus as one of the leading causes of manifestation. This trend was seen in our series as well.

Acute onset of neurological manifestations supported by radiological findings helps to establish the diagnosis of PRES. MRI findings classically include vasogenic edema in subcortical white matter predominantly in the parietal-occipital region [Figure 4]. It may also extend to the frontal, temporal, brainstem, cerebellar, cortical, and subcortical areas [Figures 5-7]. ${ }^{[2,3]}$ The presence of hemorrhage, restricted diffusion, contrast enhancement, and vasoconstriction are all compatible with the diagnosis. MRI shows T2-hyperintense images in the affected areas. ${ }^{[3]}$ About $15 \%-25 \%$ cases can show infracts and hemorrhages. ${ }^{[2,3]}$ In our series, one patient had infarction and two had hemorrhage, i.e., 30\% patients had infraction and hemorrhages. The severity is not always related to the number of cortical and subcortical areas involved ${ }^{[4]}$ More than one areas of the brain were involved in majority of the cases in our series with the involvement of brainstem in three cases. One of the studies has described PRES associated with sepsis to involve the cortical region and that with autoimmune disease the cerebellar region. ${ }^{[4]}$ Although CT scan in acute situations may help to rule out hemorrhage and ischemia, it may not always diagnose PRES; hence, suspected cases should undergo MRI. ${ }^{[5]}$

The exact pathophysiology is unknown. It is proposed that acute changes in blood pressure lead to loss of autoregulation in cerebral circulation causing hyperperfusion, endothelial damage, and vasogenic edema. The other mechanism is probably due to endothelial dysfunction leading to vasoconstriction, hypoperfusion, and subsequent cerebral ischemia and vasogenic edema. ${ }^{[2]}$

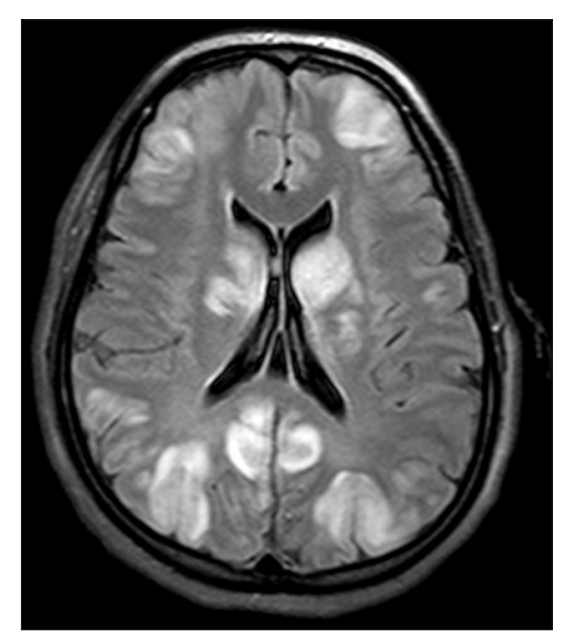

Figure 1: Brain MRI of FLAIR in 25 year old female with eclampsia demonstrating involvement of frontal and occipital lobes along with bilateral basal ganglion 


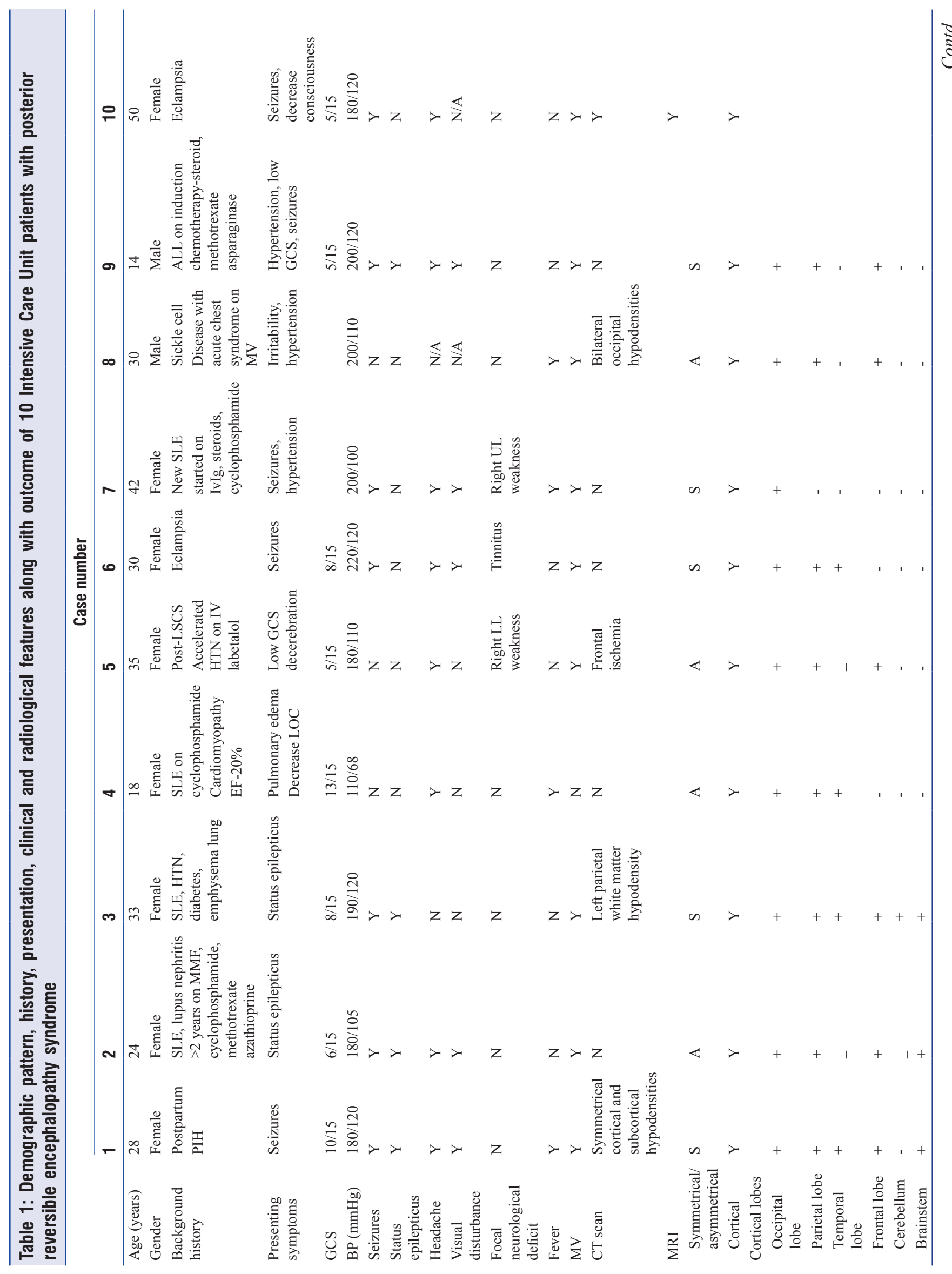


Gokhale, et al:: PRES in ICU: Case series

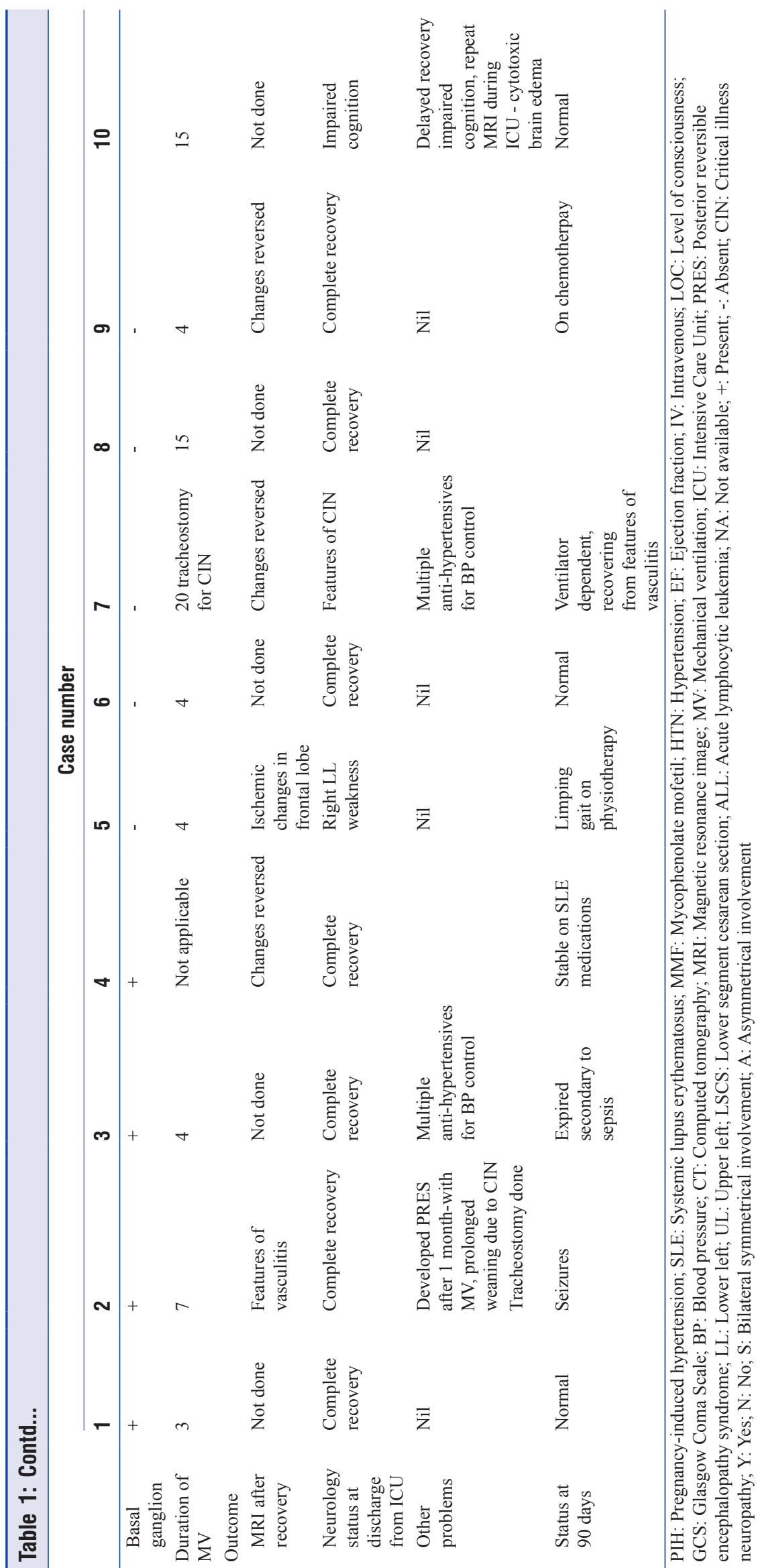




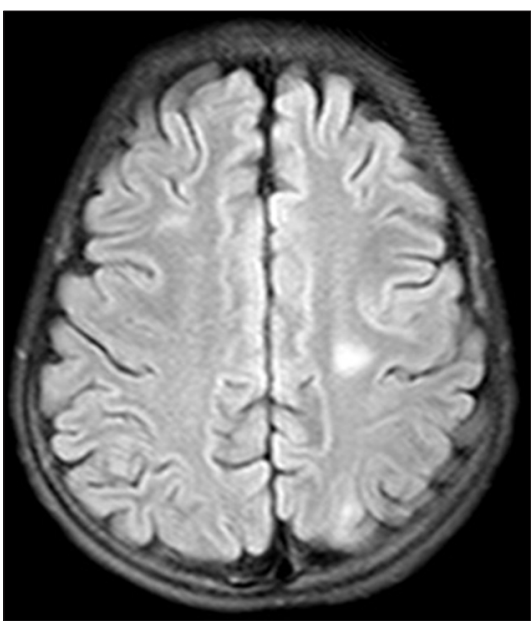

Figure 2: Brain magnetic resonance images of fluid attenuation inversion recovery sequence obtained in a 14-year-old male on induction chemotherapy with asparaginase demonstrates moderate bilateral but asymmetrical subcortical and deep white matter vasogenic edema, of parietal and occipital regions

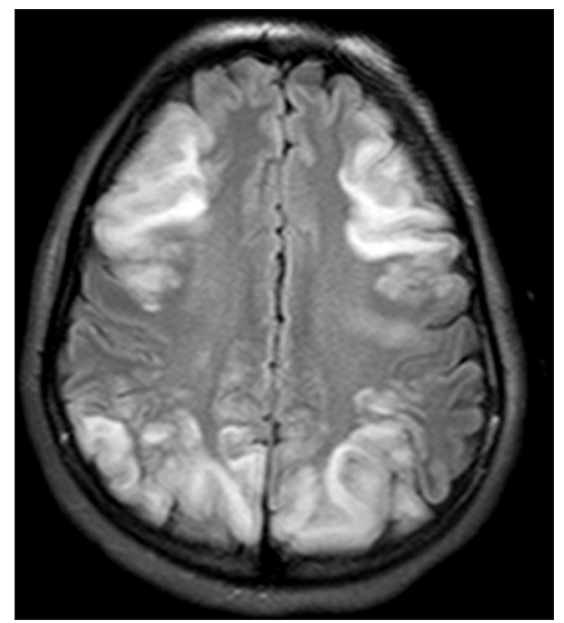

Figure 4: Brain magnetic resonance images of fluid attenuation recovery in a 25-year-old female with eclampsia demonstrates moderate bilateral and symmetrical vasogenic edema in the subcortical white matter maintaining a predominant posterior pattern

Hypertension is not found in $25 \%$ of the patients, and PRES in these situations is attributed to autoimmune or immune-mediated response. ${ }^{[2,5,6]}$ One of our cases with SLE did not have hypertension.

The etiology is varied and is associated with hypertension, autoimmune diseases, eclampsia and preeclampsia, renal diseases, and cytotoxic and immune-modulating agents. ${ }^{[2]}$ The list is ever increasing as more and more etiologies are identified with neuroimaging diagnosis. ${ }^{[3]}$

PRES shows female predominance. This was also seen in our series. It may be explained by its association with autoimmune diseases and preeclampsia/eclampsia.

Seizures are the most common presentation along with changes of encephalopathy. ${ }^{[2]}$ High degree of suspicion, prompt control

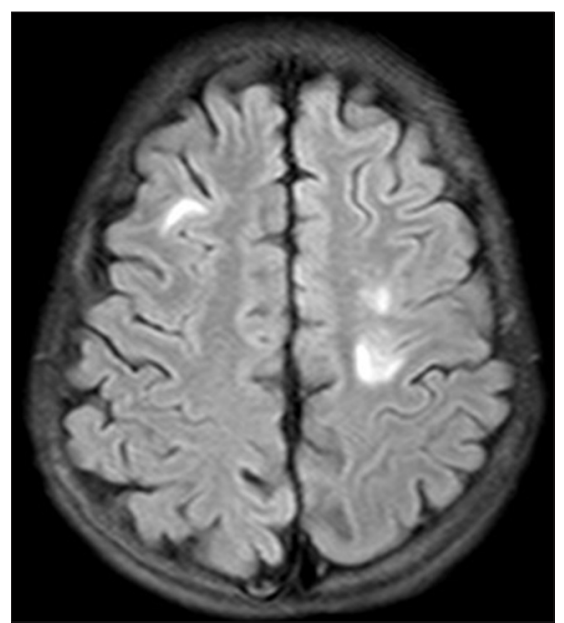

Figure 3: Brain magnetic resonance images of fluid attenuation inversion recovery sequence obtained in a 14-year-old male on induction chemotherapy with asparaginase demonstrates moderate bilateral but asymmetrical subcortical and deep white matter vasogenic edema, of temporal and parietal regions

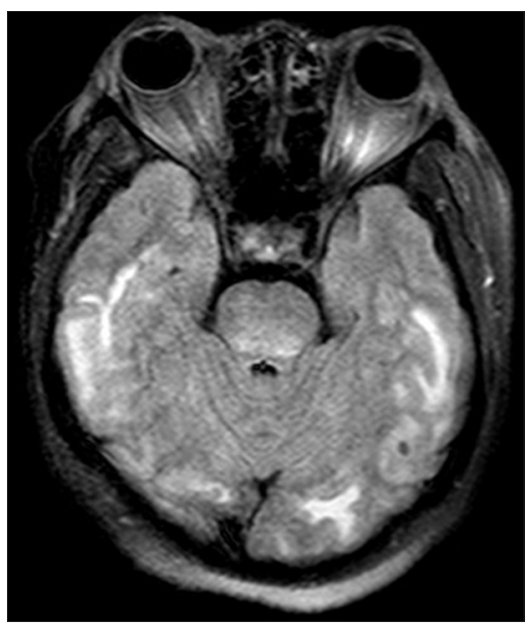

Figure 5: Brain magnetic resonance images of fluid attenuation inversion recovery sequence obtained in a 42-year-old female with systemic lupus erythematosus on immunosuppression, pulsed with steroids, demonstrates marked vasogenic edema involving the brainstem

of blood pressure, and withdrawal of the precipitating causes leads to the reversibility of clinical and radiological features. Delay can increase the morbidity and mortality with the development of brain infarcts and hemorrhages. About 15\%$20 \%$ patients may have neurological consequences; hence, the term "reversible" is questionable. ${ }^{[2,3]}$ At the time of discharge from ICU, four of our patients had residual neurological damage. At 90-day follow-up, two patients continued to have seizures and patients with frontal infarct had lower limb weakness $(30 \%)$. One patient had a repeat episode of PRES on the background of vasculitis. The MRI images in the same patient showed reversal of PRES but the development of new white matter lesions in the cerebellum and corpus callosum suggesting progression of her underlying disease. It is important to differentiate the neuropsychiatric manifestations that may 


\begin{tabular}{|c|c|}
\hline PRES cases & Observations \\
\hline Total cases & 10 \\
\hline Male:female & $2: 8$ \\
\hline Age distribution (years) & $14-42$ \\
\hline \multicolumn{2}{|l|}{ Background etiology } \\
\hline Autoimmune & 4 \\
\hline Peripartum eclampsia & 4 \\
\hline Cancer & 1 \\
\hline Others & 1 \\
\hline Medication toxicity & 4 \\
\hline \multicolumn{2}{|l|}{ Presenting symptoms } \\
\hline Headache & $9 / 10$ \\
\hline Seizures & $6 / 10$ \\
\hline Hypertension & $9 / 10$ \\
\hline Visual disturbances & $3 / 10$ \\
\hline Neurological deficits on presentation & $3 / 10$ \\
\hline MV & $9 / 10$ \\
\hline Average number of days & 8.88 \\
\hline Extubation & $7 / 9$ \\
\hline Tracheostomy & $2 / 9$ \\
\hline Death & 1 \\
\hline Residual symptoms & $3 / 10$ \\
\hline \multicolumn{2}{|l|}{ CT scan } \\
\hline Normal & $5 / 10$ \\
\hline MRI & $10 / 10$ \\
\hline Symmetrical/asymmetrical & $5 / 5$ \\
\hline Bilateral involvement & $10 / 10$ \\
\hline 1 lobe involved & $1 / 10$ \\
\hline 3 lobes involved & $6 / 9$ \\
\hline 4 lobes involved & $2 / 9$ \\
\hline Cerebellum & $2 / 9$ \\
\hline Brainstem & $3 / 10$ \\
\hline Basal ganglion & $2 / 9$ \\
\hline Hemorrhage & 3 \\
\hline MRI after recovery & $5 / 9$ \\
\hline
\end{tabular}

occur due to cerebral involvement secondary to SLE from those due to PRES. Neuroimaging is helpful. Moreover, PRES-related symptoms are more easily reversible if diagnosed early. ${ }^{[5]}$ The cause of PRES in SLE can be multifactorial, namely, underlying disease activity, hypertension, lupus nephritis, and immunosuppressant medications. ${ }^{[2,6]}$ Two patients had active disease and one was started on induction immunosuppression.

The fourth case had normal blood pressure but developed cardiomyopathy with pulmonary edema along with neurological symptoms. PRES in this situation can be explained by cytotoxic effect of SLE.[2,5]

About $24 \%-26 \%$ of patients can have residual neurological damage. Patients with frontal lobe infarction had residual left lower limb weakness at the end of 3 months. One patient presented to hospital with seizures and low level of consciousness at full-term pregnancy. She had no antenatal

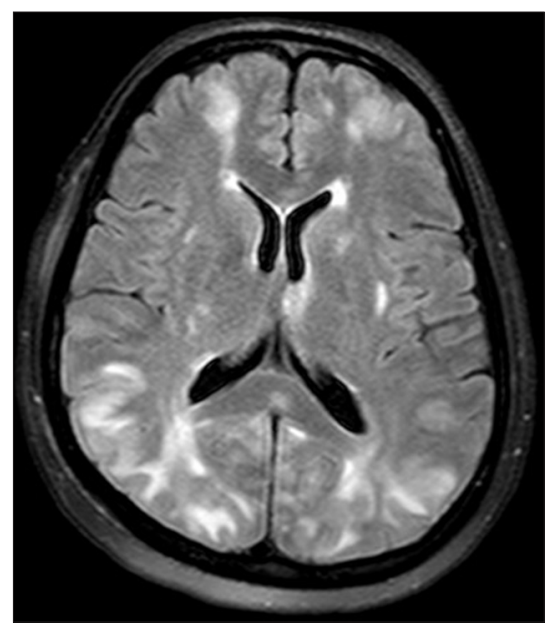

Figure 6: Brain magnetic resonance images of fluid attenuation inversion recovery sequence obtained in a 42-year-old female with systemic lupus erythematosus on immunosuppression, pulsed with steroids, demonstrates marked vasogenic edema involving the subcortical, deep, and periventricular white matter of both cerebral hemispheres, maintaining a predominant posterior pattern, along with subcortical involvement and periventricular white matter

visits and underwent emergency cesarean section. Delayed awakening and hypertension prompted neuroimaging. MRI brain showed features of PRES. The patient had impaired cognitive functions despite adequate control of blood pressure. Cytotoxic edema in multiple areas of the brain was still evident on repeat MRI. The delay in diagnosis and control of blood pressure increases the morbidity.

High incidence of PRES has been noted in eclamptics. In a series of 127 patients with eclampsia, 47 patients underwent neuroimaging for persistent hypertension and 46 were found to have features of PRES ${ }^{[6]}$ Preeclampsia is associated with alteration in blood-brain barrier, which can lead to vasogenic cerebral edema. Microangiopathic changes cause hypertension in eclampsia. PRES is attributed to lack of autoregulation, loss of blood-brain barrier, and endothelial dysfunction all of which are also the proposed etiologies for the development of eclampsia and preeclampsia. ${ }^{[2,6]}$ PRES has been reported in preeclamptics without seizures and without hypertension. ${ }^{[6,7]}$ Three of our patients had seizures postpartum and one before delivery.

Control of blood pressure is most important for reversibility of symptoms. Magnesium sulfate was used in all these patients along with the other antihypertensive medications. The recovery was related directly to control of blood pressure. Patients with postpartum eclampsia recovered faster among the entire series. Since symptoms of PRES may overlap with those of eclampsia and preeclampsia, a high degree of suspicion is required for early diagnosis and control of blood pressure. ${ }^{[8]}$ Delay in diagnosis increases the morbidity as we observed in our case.

Sickling, ischemia, and chronic anemia can contribute to vasogenic edema ${ }^{[9]}$ Endothelial dysfunction is known to occur in patients with sickle cell disease, and this could further contribute 


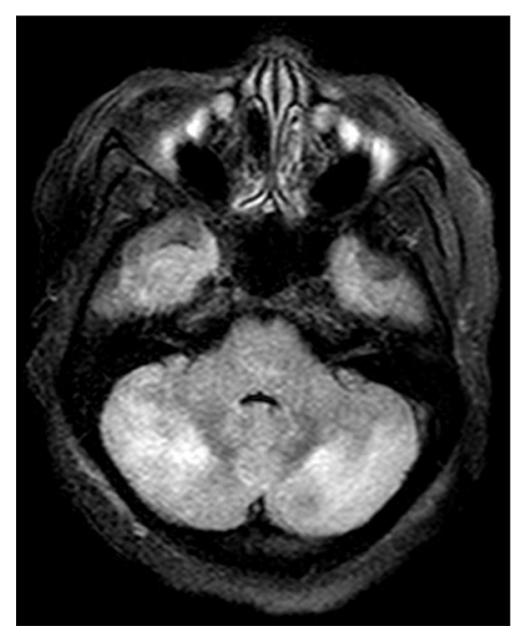

Figure 7: Brain magnetic resonance images of fluid attenuation inversion recovery sequence obtained in a 42-year-old female with systemic lupus erythematosus on immunosuppression, pulsed with steroids, demonstrates marked vasogenic edema involving the cerebellar white matter

to PRES. MRI helps to distinguish PRES from acute stroke, which is a more common neurological presentation in this group. PRES has been reported in patients with chronic anemia postblood transfusion. Abrupt increase in hemoglobin causes changes in blood viscosity and loss of hypoxemic vasodilation contributing to vasogenic edema. ${ }^{[9,10]}$ Elevated blood pressure and neuroimaging can guide the diagnosis. PRES can occur in sickle cell disease without any of the incriminating factors. ${ }^{[11]}$

PRES has been associated with multidrug chemotherapy, especially in hematological malignancies. Immunosuppressant drugs such as cyclosporine can cause endothelial damage, which can contribute to vasogenic edema secondary to release of cytokines and inflammatory agents. High-dose steroids, azathioprine, cyclophosphamide, asparaginase, and methotrexate have all been individually associated with PRES. ${ }^{[12-14]}$ Some of our patients were on one or more of these medications when diagnosed with PRES. Since primary disease control is equally important, expert help is required to modulate the medications so as to control both, the disease and symptoms of PRES.

Since patients in ICU may be ventilated and critical with a variety of co-existing problems, awareness and suspicion of PRES is important.

\section{ConcLusion}

With the increased use of neuroimaging, especially MRI, more and more cases of PRES are being diagnosed. Patients in ICU may be ventilated and critical with a variety of etiologies, and intensivists should be aware about the possibility of PRES. A high index of suspicion in patients with hypertension along with neurological manifestations should prompt a radio imaging to confirm PRES. It is well established that early diagnosis along with control of blood pressure and removal of the precipitating factors is important to decrease the morbidity and improve outcome.

\section{Declaration of patient consent}

The authors certify that they have obtained all appropriate patient consent forms. In the form the patient(s) has/have given his/her/their consent for his/her/their images and other clinical information to be reported in the journal. The patients understand that their names and initials will not be published and due efforts will be made to conceal their identity, but anonymity cannot be guaranteed.

\section{Financial support and sponsorship \\ Nil.}

\section{Conflicts of interest}

There are no conflicts of interest.

\section{ReFERENCES}

1. Hinchey J, Chaves C, Appignani B, Breen J, Pao L, Wang A, et al. A reversible posterior leukoencephalopathy syndrome. N Engl J Med 1996;334:494-500.

2. Legriel S, Pico F, Azoulay E. Understanding posterior reversible encephalopathy syndrome. In: Vincent JL, editor. Annual Update in Intensive Care and Emergency Medicine. Berlin, Heidelberg: Springer; 2011. p. 631-53.

3. Bartynski WS. Posterior reversible encephalopathy syndrome, part 1: Fundamental imaging and clinical features. AJNR Am J Neuroradiol 2008;29:1036-42.

4. Fugate JE, Rabinstein AA. Posterior reversible encephalopathy syndrome: Clinical and radiological manifestations, pathophysiology, and outstanding questions. Lancet Neurol 2015;14:914-25.

5. Liu B, Zhang X, Zhang FC, Yao Y, Zhou RZ, Xin MM, et al. Posterior reversible encephalopathy syndrome could be an underestimated variant of "reversible neurological deficits" in Systemic Lupus Erythematosus. BMC Neurol 2012;12:152.

6. Brewer J, Owens MY, Wallace K, Reeves AA, Morris R, Khan M, et al. Posterior reversible encephalopathy syndrome in 46 of 47 patients with eclampsia. Am J Obstet Gynecol 2013;208:468.e1-6.

7. Edvardsson B. Hypertensive encephalopathy and cerebral infarction. Springerplus 2014;3:741.

8. Poma S, Delmonte MP, Gigliuto C, Imberti R, Delmonte M, Arossa A, et al. Management of posterior reversible syndrome in preeclamptic women. Case Rep Obstet Gynecol 2014;2014:928079.

9. Solh Z, Taccone MS, Marin S, Athale U, Breakey VR. Neurological PRESentations in sickle cell patients are not always stroke: A Review of posterior reversible encephalopathy syndrome in sickle cell disease. Pediatr Blood Cancer 2016;63:983-9.

10. Aiyer R, Klein D, El-Sherif Y. Rare case of posterior reversible leukoencephalopathy syndrome secondary to acute chest syndrome. Case Rep Radiol 2016;2016:4346953.

11. Landais A, Lemonne N, Etienne-Julan M. Uncommon posterior reversible encephalopathy syndrome in a sickle-cell patient. J Clin Neurol 2015;11:287-8.

12. Foocharoen C, Tiamkao S, Srinakarin J, Chamadol N, Sawanyawisuth K. Reversible posterior leukoencephalopathy caused by azathioprine in systemic lupus erythematosus. J Med Assoc Thai 2006;89:1029-32.

13. Morrow SA, Rana R, Lee D, Paul T, Mahon JL. Posterior reversible encephalopathy syndrome due to high dose corticosteroids for an MS relapse. Case Rep Neurol Med 2015;2015:325657.

14. Aradillas E, Arora R, Gasperino J. Methotrexate-induced posterior reversible encephalopathy syndrome. J Clin Pharm Ther 2011;36:529-36. 